\title{
Distance Distributions for Matérn Cluster Processes with Application to Network Performance Analysis
}

\author{
Jinchuan Tang, Gaojie Chen, Justin P. Coon, David E. Simmons \\ Department of Engineering Science \\ University of Oxford \\ Parks Road, Oxford, OX1 3PJ, UK. \\ \{jinchuan.tang, gaojie.chen, justin.coon, david.simmons\}@eng.ox.ac.uk.
}

\begin{abstract}
In this work, we analyze the distance statistics corresponding to points in a Matérn cluster (offspring points) and points that do not belong to that cluster (non-offspring points). We first derive the probability density function (PDF) of the distance between an offspring point and a non-offspring point of a Matérn cluster. We then formulate the probability generating functional based on this PDF. Since many wireless networks (e.g., device-to-device (D2D) networks and cognitive radio systems) exhibit device clustering, this formalism enables us to efficiently formulate and evaluate expressions that describe the interference statistics and connection probability in clustered networks. We validate our theoretical analysis with numerical simulations, and illustrate that traditional methods of evaluating similar performance metrics (based on point process statistics instead of distance statistics) are unsuitable for use in such complex scenarios.
\end{abstract}

Index Terms-Interference, Matérn cluster process, distance probability density function, connection probability.

\section{INTRODUCTION}

Recently, stochastic geometry has become a popular modeling tool for wireless communications networks. It has played a vital role in characterizing the system-level performance of wireless networks where the nodes are placed according to random spatial point patterns. These patterns describe the nature of wireless systems statistically. With spatial point processes, performance metrics such as connection and coverage probabilities are usually formed with tractable and computable expressions. Among the spatial point processes, the Poisson point process (PPP) is very popular. It has often been used to model the positions of wireless nodes such as base stations and mobile users that are uniformly distributed in a plane. In [1] and [2], the authors presented tractable expressions for the coverage probability and average rate in the cellular downlink and uplink for both uniform and irregular network topologies. Dhillon et al. [3] considered non-uniform user equipment (UE) distributions for random cellular networks. Di Renzo [4] modeled and analyzed multi-tier millimeter wave cellular networks with PPPs.

Many wireless networking paradigms exhibit node clustering behavior. Examples of networks where this phenomenon occurs include cognitive radio networks and D2D-enabled systems. Clustering can also be caused by geographical constraints or medium access control (MAC) protocols [5]. PPP models are not capable of representing wireless networks when node clustering occurs. For this reason, Poisson cluster processes (PCPs) have attracted attention in the community. In [6], the authors showed that a PCP could accurately model active cognitive users under an exclusion region setup. They also gave a comparative study of two types of PCPs: modified Thomas cluster processes and Matérn cluster processes. Ganti and Haenggi [5] studied interference and outage in clustered wireless ad hoc networks by exploiting Matérn cluster and modified Thomas cluster processes. The authors of [7] compared the performance differences between PPP-based and modified Thomas cluster node deployments in LTE two-tier femtocell networks. In [8], the authors considered a $K$-tier heterogeneous network where the base stations of each tier are distributed by the modified Thomas cluster process and Matern cluster process to investigate the outage probability, the coverage probability, and the average achievable rate.

The analyses noted above are based on point process formalisms. When considering inhomogeneous point processes in a plane, these approaches sometimes result in complex mathematical expressions that rely on multifold integration to obtain performance results. Often, standard mathematical software packages grind to a halt when trying to evaluate such complex expressions, thus rendering the impressive theoretical results less than immediately useful in determining practical system behavior.

An alternative to using a point process formalism is to invoke distance statistics. The distribution of distances between node locations is affiliated with the interference geometry and the performance of wireless networks [9]. It is, therefore, useful and important to derive the distribution of distances between nodes in PCPs. Notably, the authors of [10] realized the importance of distance statistics for analyzing modified Thomas clusters in D2D networks and were able to conduct a performance analysis based on the distance probability density function (PDF) in that scenario. Like the modified Thomas process, the Matern cluster process is also an important process for cluster analysis. In order to understand interference statistics, arising from a Matérn cluster of interferers, observed at any point on the plane, the distribution of the distance between an offspring point (of the cluster) and a non-offspring point must be known. To the best of our knowledge, no such result has been reported in the literature before now. Knowledge of this distance distribution would reduce the 
complexity of the associated performance analysis and reduce numerical computation time.

In this paper, we address the problem alluded to above. To this end, we first derive the distance PDF between an offspring point of a Matérn cluster and a non-offspring point relative to the same cluster. We then provide a discussion of the corresponding probability generating functional (PGFL) based on this distance distribution. We also analyze the distance PDF between two offspring points in a Matérn cluster as well as the interference statistics and the connection probability for a Matérn clustered network. The simulations show that by using the distance statistics, the expressions take much less time to evaluate in a high-density network.

The rest of the paper is organized as follows. Section II begins with the discussion of the definition of a Matérn cluster process, follows with a theorem on the new distance PDF noted above, and ends by addressing the PGFL based on the distance PDF. Section III focuses on a special case study that exhibits the usefulness of the distance PDF in characterizing interference and the connection probability for two devices. Section IV documents a comparison of theoretical and simulation results. Section V concludes the paper.

\section{Distance Distribution ANALYsis}

\section{A. Matérn cluster process}

In the Matérn cluster process, the distribution of parents on two-dimensional space is a homogeneous PPP (HPPP) $\Phi_{o}=\left(N, X_{o} \mid N\right)$ with intensity $\lambda_{o}$, where $N$ is the random number of points and $X_{o} \mid N$ represents the conditionally independent and identically distributed (i.i.d.) points of $\Phi_{o}$ [11]. A realization of an HPPP on this space has an infinite number of points but is finite on any bounded window with probability tending to one. For a given observing window with area $A$ (Fig. 1), the Matérn cluster process is defined as: (i) The parents inside the window follow uniform distribution in locations and Poisson distribution with mean $A \lambda_{o}$ in number. (ii) The radii of all clusters are $R$ and the parents are the cluster centers. (iii) The offspring points uniformly spread within each cluster and obey Poisson distribution with mean $\bar{m}$ in number. The conditional PDF of an offspring point $\mathbf{u}$ inside a cluster centered at $\mathbf{x}_{O}$ is given by

$$
f\left(\mathbf{u} \mid \mathbf{x}_{o}\right)=\frac{1}{\pi R^{2}}, \text { for } r=\left\|\mathbf{u}-\mathbf{x}_{o}\right\| \leq R .
$$

From now on, each cluster consists only of offspring points. Any point that does not belong to a cluster is called a non-offspring point of that cluster. The cluster origins are distributed independently. Thus it is possible that an offspring point $\mathbf{u}_{1}$ of cluster $\mathrm{I}$ is within the radius of cluster II, but $\mathbf{u}_{1}$ is a non-offspring point of cluster II.

\section{B. A new distance distribution}

Random network performance metrics such as capacity and connection probability are related to the distances between the nodes. By providing the distance PDF, the complexity of the analysis of these metrics is shown to decrease in [10]. For

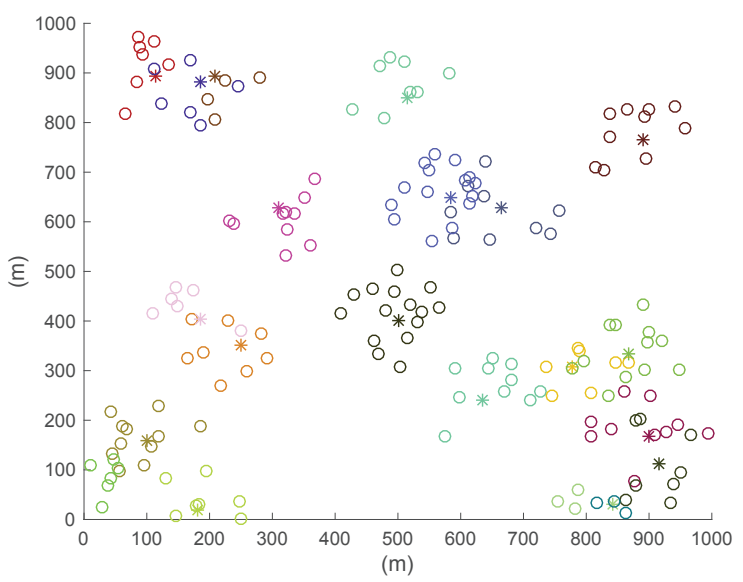

Fig. 1. The Matérn clusters where $*$ denotes the parents and $\circ$ denotes the offspring points. The parent density is 13 points per $\mathrm{km}^{2} . R=100 \mathrm{~m}$, each cluster is set to have an average of 9 offspring points.

two offspring points belonging to a Matérn cluster, the PDF of their distance $D$ is given by [12]

$$
f_{D}(d)=\frac{4 d}{\pi R^{2}}\left(\arccos \left(\frac{d}{2 R}\right)-\frac{d}{2 R} \sqrt{1-\left(\frac{d}{2 R}\right)^{2}}\right)
$$

where $0 \leq d \leq 2 R$. Interestingly, the distance distribution between an offspring point and a non-offspring point of the same cluster has not been reported in the literature. To solve this problem, traditional methods described in [5], [6] reformulate the analysis by forming distance relationships based on points and the point PDF is given by (1). This formulation is deficient in terms of the analytical complexity because the number of integrals is twice the number of integrals encountered when the problem is formulated using distance statistics (in twodimensional space). Observing that the distance distribution is of great importance to the analysis of clusters in wireless networks, we give the following theorem, which provides the distance PDF between an offspring point and a non-offspring point of a Matérn cluster.

Theorem 1. Let $R_{d}$ be a random variable representing the distance between an offspring point and a non-offspring point of a Matérn cluster with radius $R$. Conditioning on the distance $R_{y}$ from the cluster origin to the non-offspring point, the PDF of $R_{d}$ is given by

$$
f_{R_{d}}\left(r_{d} \mid R_{y}\right)=\frac{c\left(r_{d}\right)}{\pi R^{2}}
$$

where for $R_{y}>R$,

$$
\begin{gathered}
c\left(r_{d}\right)= \begin{cases}c_{1}\left(r_{d}\right), & r_{d} \in\left[R_{y}-R, R_{y}+R\right] \\
0, & \text { otherwise }\end{cases} \\
c_{1}\left(r_{d}\right)=2 r_{d} \arcsin \frac{\sqrt{4 R_{y}^{2} r_{d}^{2}-\left(R_{y}^{2}-R^{2}+r_{d}^{2}\right)^{2}}}{2 R_{y} r_{d}}
\end{gathered}
$$




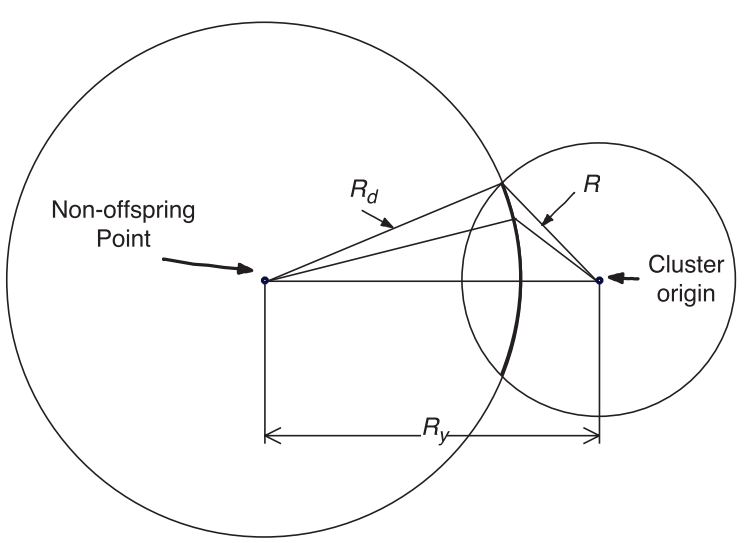

Fig. 2. The non-offspring point is outside the cluster, and a cluster encloses the arc of a circle.

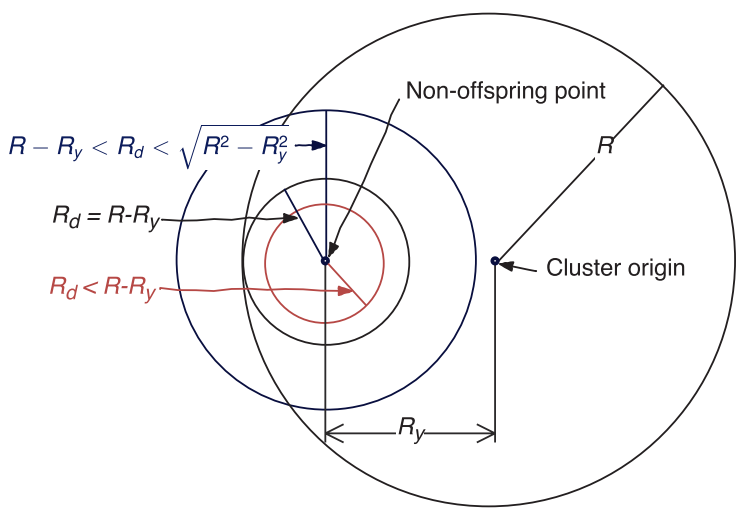

Fig. 3. The non-offspring point is inside the cluster, and a cluster encloses an arc or a circle where $R_{d}<\sqrt{R^{2}-R_{y}^{2}}$.

otherwise

$$
c\left(r_{d}\right)= \begin{cases}2 \pi r_{d}, & r_{d} \leq R-R_{y}, \\ 2 \pi r_{d}-c_{1}\left(r_{d}\right), & R-R_{y}<r_{d} \leq \sqrt{R^{2}-R_{y}^{2}}, \\ c_{1}\left(r_{d}\right), & \sqrt{R^{2}-R_{y}^{2}}<r_{d} \leq R+R_{y}, \\ 0, & \text { otherwise. }\end{cases}
$$

Proof: When $R_{y}>R$, the non-offspring point is outside the Matérn cluster. The possible locations of the offspring point that have the same distance to the non-offspring point are on the circular arc of a circle that is enclosed by the cluster as shown in Fig. 2. When $R_{d}$ is increasing from $R_{y}-R$ to $R_{y}+R$, the cluster can be filled with an infinite number of nonoverlapping arcs, and each arc represents a class of points that have the same distance to the non-offspring point. Hence by calculating the arc length with (4), the corresponding density for $R_{d}$ is achieved in (3).

When $0<R_{y} \leq R$, the non-offspring point is inside the Matérn cluster. The possible locations of the offspring point that have the same distance to a non-offspring point could be on: (i) the circle centered at the non-offspring point when $R_{d} \leq R-R_{y}$ as depicted in Fig. 3; (ii) the circular arc of

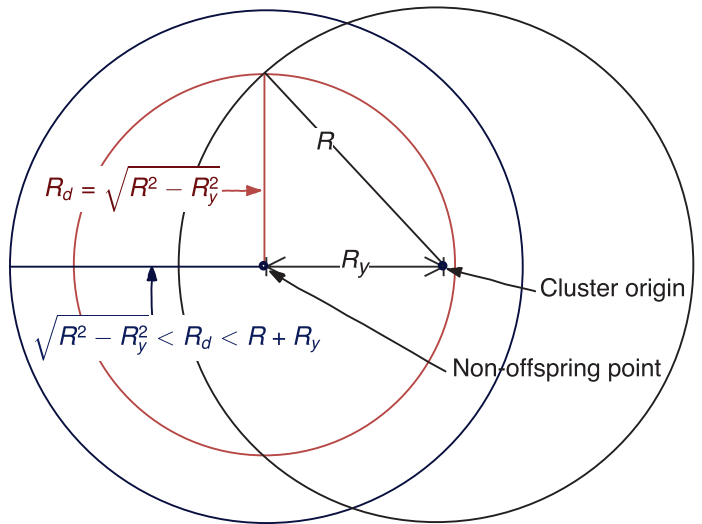

Fig. 4. The non-offspring point is inside the cluster, and a cluster encloses an arc or a circle where $R_{d}>\sqrt{R^{2}-R_{y}^{2}}$.

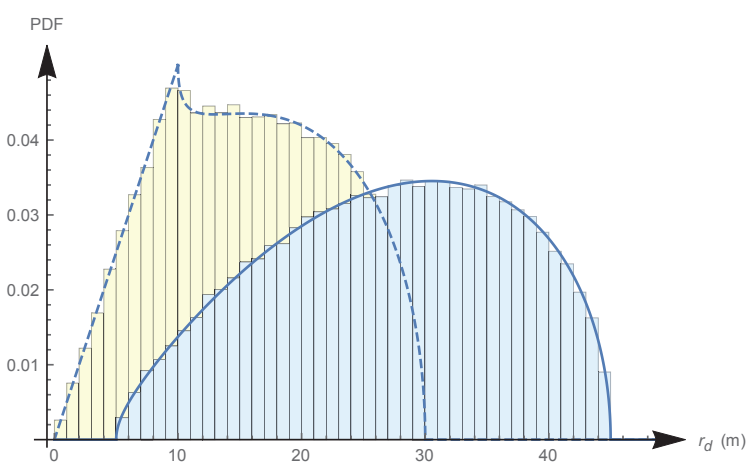

Fig. 5. The plot of (3) where the dashed line represents the PDF given by $R_{y}=10 \mathrm{~m}$ and $R=20 \mathrm{~m}$, while the solid line represents the PDF given by $R_{y}=25 \mathrm{~m}$ and $R=20 \mathrm{~m}$. The bins represent the simulation results for both PDFs.

a circle with radius $R_{d}$ that is enclosed by the cluster with radius $R$ as seen in Figs. 3 and 4 .

The former is the perimeter of a circle with radius $R_{d}$, which is the first case of (5). The latter belongs to the second and third cases of (5). $\sqrt{R^{2}-R_{y}^{2}}$ is the boundary to decide if the circular arc enclosed by the cluster is the major or minor arc of a circle with radius $R_{d}$.

When $R_{y}=0$, the non-offspring point is overlapping with the cluster origin. The possible locations of the offspring point that have the same distance to the non-offspring point form a circle with radius $R_{d}$ centered at the cluster origin. It belongs to the first case in (5). Thus we conclude our proof.

Fig. 5 shows two examples of Theorem 1. Both examples are given the same cluster radius $R$ but different values of $R_{y}$ to demonstrate both (4) and (5). The solid line shows that (4) offers a smooth concave curve while the dashed line shows signs of transitions in (5). The tip and the inflection point on the dashed line are the transition points from case one to two and case two to three in (5), respectively. The simulation results have shown in the examples a good match to the theoretical values. 


\section{Probability generating functional (PGFL)}

A Matérn cluster process is a type of Neyman-Scott process [5], hence it follows the definition of the probability generating functional (PGFL) for the Neyman-Scott processes. For a given function $g: \mathbb{R}^{2} \rightarrow[0,1]$ and $\int_{\mathbb{R}^{2}}|1-g(\mathbf{x})| \mathrm{d} \mathbf{x}<\infty$, where $\mathbf{x}=\left(x_{1}, x_{2}\right)$ denotes the Cartesian coordinate of a point in the two-dimensional plane, the PGFL of a Neyman-Scott process $\Phi$ is given by [5]

$$
\begin{aligned}
& \mathbb{G}_{\Phi}(g)=\mathbb{E}_{\Phi}\left[\prod_{\mathbf{x} \in \Phi} g(\mathbf{x})\right]= \\
& \exp \left\{\lambda \int_{0}^{2 \pi} \int_{0}^{\infty}\left[\eta\left(\int_{\mathbb{R}^{2}} g(\mathbf{x}+\mathbf{b}) f(\mathbf{x} \mid \mathbf{0}) \mathrm{d} \mathbf{x}\right)-1\right] r \mathrm{~d} r \mathrm{~d} \theta\right\}
\end{aligned}
$$

where $\mathbf{b}=(r \cos (\theta), r \sin (\theta)), \eta(z)$ is the moment-generating function of a Poisson random variable which describes the distribution of the number of offspring points in a cluster, and $f(\mathbf{x} \mid \mathbf{0})$ is the conditional PDF (1) originated at $\mathbf{0}=(0,0)$ for a Matérn point process with radius $R$.

Noticing that $R_{y}=r$ and having the distance PDF given by Theorem 1, we see that if $g(\mathbf{x})$ is equal to a distance function $g_{1}(|| \mathbf{x}||)$, the PGFL is given by

$$
\begin{aligned}
& \mathbb{G}_{\Phi}\left(g_{1}\right) \\
& =\exp \left\{2 \pi \lambda \int_{0}^{\infty}\left[\eta\left(\int_{\mathbb{D}} g_{1}(x) f_{R_{d}}(x \mid r) \mathrm{d} x\right)-1\right] r \mathrm{~d} r\right\},
\end{aligned}
$$

where $\mathbb{D}=\left[[r-R]^{+}, r+R\right]$ is the domain for the distance PDF in Theorem 1, and $[r-R]^{+}=\max (0, r-R)$. Notably, the number of integrals is reduced from 4 integrals in (6) to 2 integrals in (7).

\section{APPLICATIONS}

To understand how to use the distance statistics for network performance analysis, we provide a case study.

\section{A. System model}

To exploit the distance PDFs for cluster interference analysis, we focus on a special case study where the simultaneously active transmitting nodes, which use the same channel resource, are distributed according to Matérn cluster processes. We assume that the intensity of the cluster centers is $\lambda_{o}$, the mean of the transmitting nodes in a cluster is $\bar{m}$ and the corresponding receiving nodes are uniformly distributed in the clusters. Therefore, a typical receiving node inside a cluster experiences interference from the undesired transmitting nodes, which are active simultaneously. To simplify the analysis, we choose the path loss model $^{1} l(\|\mathbf{x}-\mathbf{y}\|)=(\lambda / 4 \pi)^{2}\|\mathbf{x}-\mathbf{y}\|^{-\alpha}$, which is given by [14], where $\lambda$ represents the carrier wavelength and $\|\mathbf{x}-\mathbf{y}\|$ is the distance between a node at point $\mathbf{x}$ and a node at point $\mathbf{y}$. We assume that the fading of

\footnotetext{
${ }^{1}$ Other path-loss models such as empirically based models and log-normal shadowing [13] are also applicable to our analysis, but they are out of the scope of this paper.
}

each channel is Rayleigh distributed. The gain due to coding, transmitting antenna and receiving antenna is $G_{e}$. Hence, $G_{e} l(\|\mathbf{x}-\mathbf{y}\|)$ is the link gain. The power of a typical transmitting node is $P_{d}$.

\section{B. Interference analysis}

1) Interference analysis in general: The total interference power measured at a receiving node at point $\mathbf{x}$ is given by

$$
I(\mathbf{x})=\sum_{\mathbf{y} \in \Phi} P_{r_{\mathbf{y}}}(\mathbf{x})=\sum_{\mathbf{y} \in \Phi} G_{e} \ell(\|\mathbf{x}-\mathbf{y}\|)\left|h_{\mathbf{x y}}\right|^{2} P_{d},
$$

where $\Phi$ is a point process, which consists of simultaneously active transmitting nodes, and $P_{r_{\mathbf{y}}}(\mathbf{x})$ is the interference received at point $\mathbf{x}$ from one such node at point $\mathbf{y}$.

Considering the interference as a shot noise process and identifying the fading terms $h_{\mathbf{x y}}$ as i.i.d. Rayleigh fading $h$, the Laplace transform of the interference is defined as [6]

$$
\begin{aligned}
\mathcal{L}_{I}(s) & =\mathbb{E}_{\Phi, h}[\exp (-s I(\mathbf{x}))] \\
& =\mathbb{E}_{\Phi}\left[\prod_{\mathbf{y} \in \Phi} \frac{1}{1+s G_{e} \ell(\|\mathbf{x}-\mathbf{y}\|) P_{d}}\right] .
\end{aligned}
$$

In our system, $\Phi$ causes two independent types of interference: intra-cluster and inter-cluster interference. Given a receiving node inside a cluster, the former describes the interference to the receiving node coming from the simultaneously active transmitting nodes in the same cluster, while the latter accounts for the interference coming from the simultaneously active transmitting nodes from other clusters [15].

The following two subsections study the intra-cluster and inter-cluster interference analysis respectively. Each subsection begins with the traditional analysis based on point coordinates and ends up with the proposed analysis based on distance.

2) Intra-cluster interference:

a) Analysis based on point coordinates: Assuming that a typical receiver is at the origin and its cluster is centered at point $\mathbf{c}$, the Laplace transform of the intra-cluster interference based on point coordinates and the point distribution (1) is given by

$$
\mathcal{L}_{I}^{\text {intra }}(s)=\int_{\mathbb{R}^{2}} \mathcal{L}_{I}^{\text {intra }}(s \mid \mathbf{c}) f(\mathbf{c} \mid \mathbf{0}) \mathrm{d} \mathbf{c},
$$

where

$$
\begin{aligned}
& \mathcal{L}_{I}^{\text {intra }}(s \mid \mathbf{c}) \\
& =\exp \left\{-(\bar{m}-1) \int_{\mathbb{R}^{2}} \frac{g(\mathbf{a}+\mathbf{c})}{s^{-1}+g(\mathbf{a}+\mathbf{c})} f(\mathbf{a} \mid \mathbf{0}) \mathrm{d} \mathbf{a}\right\},
\end{aligned}
$$

with $g(\mathbf{x})=G_{e} P_{d} \ell(\|\mathbf{x}\|)$. As observed from above, the Laplace transform of all interferers in a cluster can be characterized by a typical interferer with an offset a from a cluster center c. $\mathcal{L}_{I}^{\text {intra }}(s \mid \mathbf{c})$ is the Laplace transform of intracluster interference conditioning on the cluster center c. By marginalizing over the possible locations of the cluster center c, $\mathcal{L}_{I}^{\text {intra }}(s)$ is achieved. 
b) Analysis based on distance: The PDF of distance $D$ between the receiving node and interferer, which are independently and uniformly distributed in a circular area with radius $R$ is given by (2); hence the distance version of intra-cluster interference in the PCP is written as

$$
\mathcal{L}_{I}^{\text {intra }}(s)=\exp \left(-(\bar{m}-1) \int_{0}^{2 R} \frac{g_{1}(x)}{s^{-1}+g_{1}(x)} f_{D}(x) \mathrm{d} x\right),
$$

where $g_{1}(x)=G_{e} P_{d} \ell(x)$. The Laplace transform of the intracluster interference conditioning on the distance $v$ between the receiver and its cluster center is given by

$$
\begin{aligned}
& \mathcal{L}_{I}^{\text {intra }}(s \mid v) \\
& =\exp \left\{-(\bar{m}-1) \int_{[v-R]^{+}}^{v+R} \frac{g_{1}(x)}{s^{-1}+g_{1}(x)} f_{R_{d}}(x \mid v) \mathrm{d} x\right\} .
\end{aligned}
$$

\section{3) Inter-cluster interference:}

a) Analysis based on point coordinates: The Laplace transform of inter-cluster interference based on point distributions is given by

$$
\mathcal{L}_{I}^{\text {inter }}(s)=\exp \left(-\lambda_{o} \int_{\mathbb{R}^{2}}\left[1-\xi_{1}(s, \mathbf{c})\right] \mathrm{d} \mathbf{c}\right),
$$

where $\xi_{1}(s, \mathbf{c})=\exp \left\{-\bar{m} \int_{\mathbb{R}^{2}} \frac{g(\mathbf{a}+\mathbf{c})}{s^{-1}+g(\mathbf{a}+\mathbf{c})} f(\mathbf{a} \mid \mathbf{0}) \mathrm{d} \mathbf{a}\right\}$.

b) Analysis based on distance: Applying (7), the Laplace transform of inter-cluster interference based on the distance distribution is written as

$$
\mathcal{L}_{I}^{\text {inter }}(s)=\exp \left(-2 \pi \lambda_{o} \int_{0}^{\infty}\left[1-\xi_{2}(s, v)\right] v \mathrm{~d} v\right),
$$

where $\xi_{2}(s, v)=\exp \left\{-\bar{m} \int_{[v-R]}^{v+R}+\frac{g_{1}(x)}{s^{-1}+g_{1}(x)} f_{R_{d}}(x \mid v) \mathrm{d} x\right\}$.

As a result of the above discussion, we have successfully built up the methods for interference analysis based on the distribution of distance.

\section{Connection probability analysis}

The connection probability of a link is the likelihood that the actual SINR of the link is higher than a predetermined threshold $\beta=2^{t}-1$. The parameter $t=R_{b} / B$ is the predefined link spectrum efficiency for a node, $R_{b}$ is the target rate of the link in bit/s, and $B$ is the transmission bandwidth. Giving the distance $d$ between a typical transmitting node and receiving node and considering that the noise and interference are independent of each other [16], the connection probability of the typical transmitting node conditioned on the distance $v$ between the receiver and its cluster center is written as

$$
\begin{aligned}
& p(d \mid v)=\exp \left(-\frac{\beta N_{0}}{g_{1}(d)}\right) \mathcal{L}_{I}\left(\frac{\beta}{g_{1}(d)} \mid v\right) \\
& =\exp \left(-\frac{\beta N_{0}}{g_{1}(d)}\right) \mathcal{L}_{I}^{\text {intra }}\left(\frac{\beta}{g_{1}(d)} \mid v\right) \mathcal{L}_{I}^{\text {inter }}\left(\frac{\beta}{g_{1}(d)}\right),
\end{aligned}
$$

TABLE I

SYSTEM PARAMETERS.

\begin{tabular}{ll}
\hline Parameter & value \\
\hline Bounded area & $4 \mathrm{~km} \times 4 \mathrm{~km}$ \\
Observing window area $A$ & $2 \mathrm{~km} \times 2 \mathrm{~km}$ \\
Transmission bandwidth $B$ & $20 \mathrm{MHz}$ \\
Duplex mode & Half duplex \\
Thermal noise power density & $-174 \mathrm{dBm} / \mathrm{Hz}$ \\
Cluster radius $R$ & $12.5 \mathrm{~m}, 50 \mathrm{~m}, 100 \mathrm{~m}$ \\
Transmitting power level $P_{d}$ & $23 \mathrm{dBm}$ \\
Coding gain & $0 \mathrm{~dB}$ \\
Transmitting antenna gain & $0 \mathrm{dBi}$ \\
Receiving antenna gain & $0 \mathrm{dBi}$ \\
Transceiver noise figure & $9 \mathrm{~dB}$ \\
Link spectrum efficiency & $0.05 \mathrm{bps} / \mathrm{Hz}$ \\
Carrier frequency & $2 \mathrm{GHz}$ \\
Pathloss exponent & 2.7 \\
Simulation iterations & $10^{4}$ \\
\hline
\end{tabular}

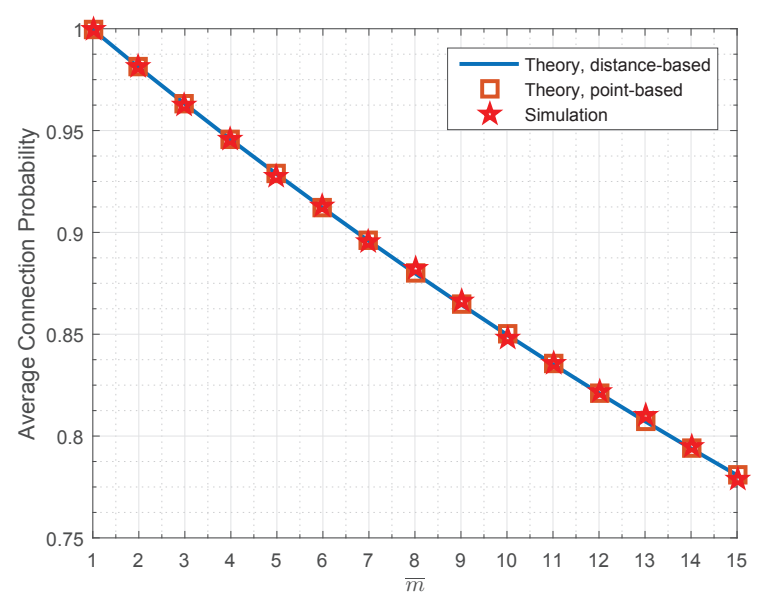

Fig. 6. The average connection probability of a typical node in the Matern cluster of the network vs. an average of $\bar{m}$ transmitting nodes inside each cluster. $\lambda_{o}$ is 0.5 points per $\mathrm{km}^{2}$, and $R=12.5 \mathrm{~m}$. The average number of clusters in the observing window is $A \lambda_{o}=2$.

where $N_{0}$ is the thermal noise power at the receiving node, and the final term is because the intra-cluster interference and intercluster interference are also independent. Eqs. (13) and (15) are chosen respectively for (16). The connection probability of the typical transmitting node is given by

$$
p=\int_{0}^{R} \int_{0}^{v+R} p\left(r_{d} \mid v\right) f_{R_{d}}\left(r_{d} \mid v\right) \frac{2 v}{R^{2}} \mathrm{~d} r_{d} \mathrm{~d} v .
$$

\section{Simulation Results}

In this section, we provide simulation results to validate the method detailed above. The parameters used in the simulation are given in Table I. The simulation results are obtained by averaging over $10^{4}$ independent Monte Carlo trials. To combat boundary effects, we use the measurements recorded in an observation window with area $A$ centered in a bounded area. The average number of clusters in the observation window is given by $A \lambda_{o}$.

Fig. 6 shows that for a given average number of clusters, the connection probability of a network node decreases as 


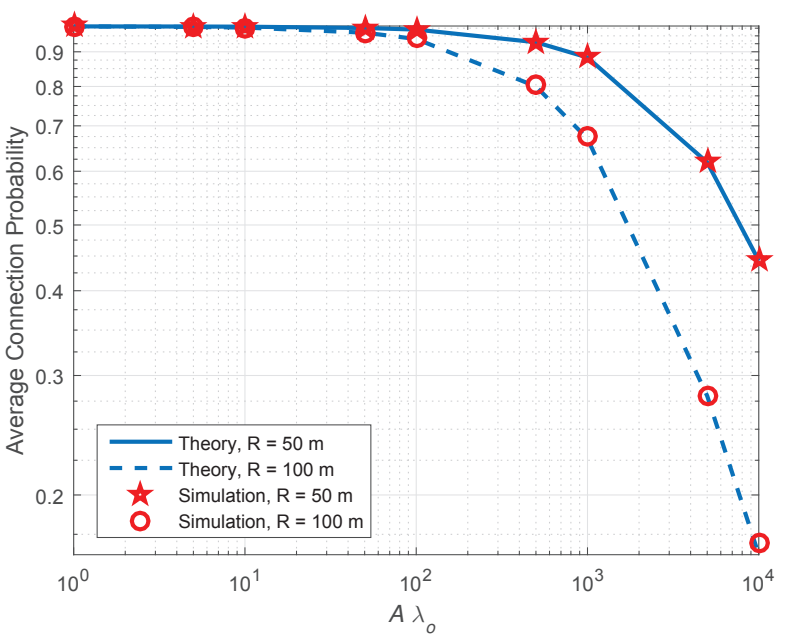

Fig. 7. The average connection probability of a typical node vs. the average number of clusters in the observing window: $A \lambda_{o}$. The average number of transmitting nodes in a cluster is $\bar{m}=2$.

the average number of transmitting nodes in each cluster $\bar{m}$ increases. The performance of proposed analysis based on distance matches well with both the traditional analysis based on point coordinates and the simulation results. The numerical integrations of proposed analysis based on distance with (17) and traditional analysis based on points were computed using Mathematica with the same working precision on an Intel Core i7-4790 PC with 16 GB memory. Under the 3 digits precision goal, our proposed analysis spent approximately $23 \%$ less time than the traditional analysis to produce each point in the figure.

Fig. 7 illustrates that the average connection probability of a typical network node decreases drastically as the cluster density $\lambda_{o}$ increases. For a given average number of nodes $\bar{m}$ within the cluster, the larger the radius of the cluster, the smaller average connection probability the system will have. It is because the radius is increasing so that the transmitting and receiving nodes have a larger separation on average and the interferers are also closer to the receiving node (on average). For a sample $A \lambda_{o} \in\left[10^{0}, 10^{2}\right)$, both the proposed analysis based on distance and traditional analysis based on point coordinates finished the calculations within 1.5 hours. However, when $A \lambda_{o}$ is getting larger, the traditional analysis slowed down to produce the results. For example, when $R=50 \mathrm{~m}$ and $A \lambda_{o}=5 \times 10^{3}$, the proposed analysis cost around 1 hour and the traditional analysis spent around 11 hours. When $R=100 \mathrm{~m}$, for a sample $A \lambda_{o} \in\left[10^{2}, 10^{4}\right]$, the traditional analysis produced no result after 24 hours while the proposed analysis finished within 2.5 hours. Therefore, our proposed analysis based on distance is of significant advantage over the traditional analysis in terms of time to achieve analytical results in a high-density network.

\section{CONCLUSION}

In this paper, we formulated the distance PDF between an offspring point and a non-offspring point of a Matérn cluster.
Based on this, we have characterized the PGFL, interference statistics and connection probability of a typical network node. By doing so, the number of integrals is halved compared to the traditional point-based method. Our method consumes much less computational time in a high-density network. Therefore, the proposed methods will facilitate performance analysis for wireless networks such as cognitive radio systems and D2D communications for $5 \mathrm{G}$ and beyond.

\section{ACKNOWLEDGMENT}

The authors wish to acknowledge the support of EPSRC grant EP/N002350/1.

\section{REFERENCES}

[1] J. G. Andrews, F. Baccelli, and R. K. Ganti, "A tractable approach to coverage and rate in cellular networks," IEEE Trans. Commun., vol. 59 no. 11, pp. 3122-3134, Nov. 2011.

[2] T. D. Novlan, H. S. Dhillon, and J. G. Andrews, "Analytical modeling of uplink cellular networks," IEEE Trans. Wireless Commun., vol. 12, no. 6, pp. 2669-2679, Jun. 2013.

[3] H. S. Dhillon, R. K. Ganti, and J. G. Andrews, "Modeling nonuniform UE distributions in downlink cellular networks," IEEE Wireless Commun. Lett., vol. 2, no. 3, pp. 339-342, Jun. 2013.

[4] M. Di Renzo, "Stochastic geometry modeling and analysis of multitier millimeter wave cellular networks," IEEE Trans. Wireless Commun. vol. 14, no. 9, pp. 5038-5057, Sep. 2015.

[5] R. K. Ganti and M. Haenggi, "Interference and outage in clustered wireless ad hoc networks," IEEE Trans. Info. Theory, vol. 55, no. 9, pp. 4067-4086, Sep. 2009.

[6] C.-h. Lee and M. Haenggi, "Interference and outage in Poisson cognitive networks," IEEE Trans. Wireless Commun., vol. 11, no. 4, pp. 1392 1401, Apr. 2012.

[7] Z. Jakó and G. Jeney, "Outage probability in Poisson-cluster-based LTE two-tier femtocell networks," Wireless Commun. Mob. Comput., vol. 15, no. 18, pp. 2179-2190, Jun. 2015.

[8] Y. J. Chun, M. O. Hasna, and A. Ghrayeb, "Modeling heterogeneous cellular networks interference using poisson cluster processes," IEEE J. Sel. Areas Commun., vol. 33, no. 10, pp. 2182-2195, Oct. 2015.

[9] F. Voss, C. Gloaguen, F. Fleischer, and V. Schmidt, "Distributional properties of Euclidean distances in wireless networks involving road systems," IEEE J. Sel. Areas Commun., vol. 27, no. 7, pp. 1047-1055, Sep. 2009.

[10] M. Afshang, H. Dhillon, and P. Chong, "Modeling and performance analysis of clustered device-to-device networks," IEEE Trans. Wireless Commun., vol. 15, no. 7, pp. 4957-4972, Jul. 2016.

[11] R. L. Streit, Poisson point processes: imaging, tracking, and sensing. Virginia, USA: Springer Science \& Business Media, 2010.

[12] H. C. Tuckwell, Elementary applications of probability theory. London, UK: Chapman \& Hall, 1995.

[13] B. Black, P. DiPiazza, B. Ferguson, D. Voltmer, and F. Berry, Introduction to Wireless Systems, 1st ed. Upper Saddle River, NJ: Prentice Hall Press, 2008.

[14] E. McCune, Practical digital wireless signals, ser. The Cambridge RF and Microwave Engineering Series. Cambridge, UK: Cambridge Uni. press, 2013.

[15] G. Chen and J. A. Chambers, "Exact outage probability analysis for cooperative af relay network with relay selection in presence of intercell interference," Electronics Letters, vol. 48, no. 21, pp. 1346-1347, Oct. 2012

[16] M. Haenggi and R. K. Ganti, "Interference in large wireless networks," Found. Trends Netw., vol. 3, no. 2, pp. 127-248, Feb. 2009. 